\title{
Correction to: Association Between Health Literacy and Medication Adherence Among Hispanics with Hypertension
}

\author{
Maichou Lor $^{1} \cdot$ Theresa A. Koleck $^{1} \cdot$ Suzanne Bakken ${ }^{1,2,3} \cdot$ Sunmoo Yoon ${ }^{1} \cdot$ Ann-Margaret Dunn Navarra ${ }^{4}$
}

Published online: 29 April 2019

(C) W. Montague Cobb-NMA Health Institute 2019

\section{Correction to: Journal of Racial and Ethnic Health Disparities pp 1-8 https://doi.org/10.1007/s40615-018-00550-z}

Please note the following correction in the second paragraph of the Results section of this article, in the sentences that read, "Table 2 shows the association of health literacy level, as well as each of the included covariates with adherence. Adequate health literacy was associated with a higher adherence score, i.e., "better" adherence $(b=0.0572, p$ value $\left.=0.002, R^{2}=0.008\right)$, as compared to inadequate health literacy levels."
In the second of these two sentences, " $b=0.0572$ " should read "b $=0.572$.

Publisher's Note Springer Nature remains neutral with regard to jurisdictional claims in published maps and institutional affiliations.

The online version of the original article can be found at https://doi.org/ 10.1007/s40615-018-00550-z

Ann-Margaret Dunn Navarra amd363@nyu.edu

1 School of Nursing, Columbia University, New York City, NY, USA

2 Biomedical Informatics, Columbia University, New York City, NY, USA

3 Data Science Institute, Columbia University, New York City, NY, USA

4 Rory Meyers College of Nursing, New York University, 433 First Avenue, New York City, NY 10010, USA 